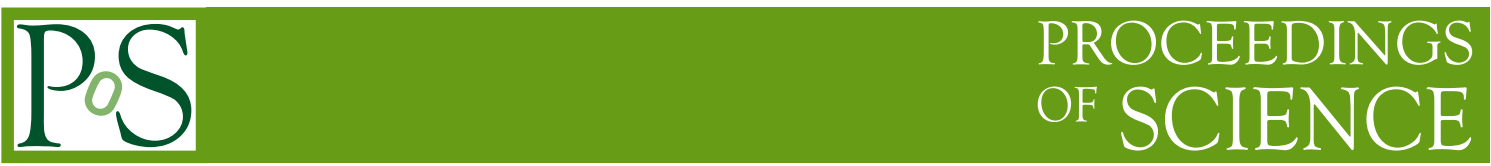

\title{
Strange hadron effective temperatures in relativistic nuclear collisions
}

Oana Ristea, ${ }^{a, *}$ Cătălin Ristea,,${ }^{a, b}$ Alexandru Jipa, ${ }^{a}$ Ionel Lazanu, ${ }^{a}$ Marius Călin ${ }^{a}$ and Tiberiu Eșanu ${ }^{a, c}$

${ }^{a}$ Faculty of Physics, University of Bucharest,

Bucharest-Măgurele, România

${ }^{b}$ Institute of Space Science,

Bucharest-Măgurele, România

${ }^{c}$ National Institute of Nuclear Physics and Engineering Horia Hulubei

Bucharest-Măgurele, România

E-mail: oana.ristea@unibuc.ro, catalin.ristea@spacescience.ro

The effective temperatures of $K^{ \pm}, \phi, \Omega^{-}$and $\bar{\Omega}^{+}$produced in Au+Au collisions at RHIC-BES energies are presented. Energy dependence of the kaon effective temperature is approximately constant at lower RHIC-BES energies, similar with previous SPS results and could indicate the onset of deconfinement in this energy range. The centrality dependence of $T_{K^{ \pm}}$showed that the charged kaon effective temperature increases at higher BES energies and also from peripheral to central collisions. The $\phi$ and $\Omega$ effective temperatures increase with energy and no plateau-like behaviour is evidenced by the data.

*** The European Physical Society Conference on High Energy Physics (EPS-HEP2021), ***

*** 26-30 July $2021 * * *$

*** Online conference, jointly organized by Universität Hamburg and the research center DESY ***

${ }^{*}$ Speaker 


\section{Introduction}

One of the main goals of the relativistic nuclear collisions studies is to investigate the hot and dense nuclear matter produced in these collisions. In such extreme conditions of temperature and energy density a phase-transition from the hadronic matter to a deconfined state of strongly interacting matter [1] can occur.

Strange and multi-strange hadrons can provide valuable information related to the properties of the created system and the onset of deconfinement. An enhanced production of strange particles, relative to $\mathrm{p}+\mathrm{p}$, was suggested as a signal of the QGP formation [2].

\section{Results}

It has been observed in heavy-ion experiments that the invariant transverse momentum distributions in $\mathrm{A}+\mathrm{A}$ collisions generally show in the low $p_{T}$ region an exponential shape in $m_{T}-m_{0}$, where $m_{0}$ is particle mass and $m_{T}=\sqrt{p_{T}^{2}+m^{2}}$ is the transverse mass. For a $p_{T}$ spectrum with an exponential shape, one can parameterize it as follows:

$$
\frac{1}{2 \pi p_{T}} \frac{d^{2} N}{d p_{T} d y}=\frac{d N / d y}{2 \pi T\left(T+m_{0}\right)} \cdot \exp \left(-\frac{m_{T}-m_{0}}{T}\right)
$$

where the particle yield, $d N / d y$, and the inverse slope parameter or the effective temperature, $T$, are parameters that can be extracted from fits to the data.

The $p_{T}$ distributions of positive and negative kaons produced in $\mathrm{Au}+\mathrm{Au}$ collisions at RHICBES energies $\left(\sqrt{s_{N N}}=7.7,11.5,14.5,19.6,27\right.$ and $\left.39 \mathrm{GeV}\right)[3]$ and of $\Omega^{-}, \bar{\Omega}^{+}, \phi$ produced in $\mathrm{Au}+\mathrm{Au}$ collisions at $\sqrt{s_{N N}}=7.7,11.5,19.6,27$ and $39 \mathrm{GeV}$ [4] were fitted using Eq. 1. The energy dependence of the $K^{ \pm}, \Omega^{-}, \bar{\Omega}^{+}, \phi$ effective temperatures is presented in Figure 1 . These results were compared with previous results from AGS, SPS, RHIC and LHC experiments $[6-12,16,18]$.

The kaon effective temperature increases at AGS energies and from higher RHIC energies up to LHC energy, whereas they are almost constant in the SPS/RHIC-BES energies. The constant temperature region in this dependence can signal a first-order phase transition to deconfined matter. It is considered that such a behaviour may arise from the modification of the equation of state of the matter due to the hadronic-deconfined phase transition [5].

It can be seen from the figure that $T_{\phi}, T_{\Omega}$ and $T_{\bar{\Omega}^{+}}$increases with increasing energy showing no plateau-like behaviour. These results at RHIC-BES energies are consistent within error bars to what was observed at other similar energies.

The centrality dependence of the charged kaon effective temperatures is shown in Figure 2. The STAR data from $\mathrm{Au}+\mathrm{Au}$ collisions have the following centrality classes: 0-5\%, 5-10\%, 10-20\%, $20-30 \%, 30-40 \%, 40-50 \%, 50-60 \%, 60-70 \%$ and $70-80 \%$. The number of participating nucleons, $N_{\text {part }}$, as a measure of system size, corresponding to these centralities is taken from [3]. For all energies, the effective temperatures for charged kaons increase with increasing $N_{\text {part }}$. This indicates an increase of radial flow from peripheral to central collisions [19]. Due to an increase in the radial transverse flow, the $p_{T}$ spectra are flattened and the extracted inverse slopes are larger. Therefore, $T$ is sensitive to both the thermal and transverse collective motion. 

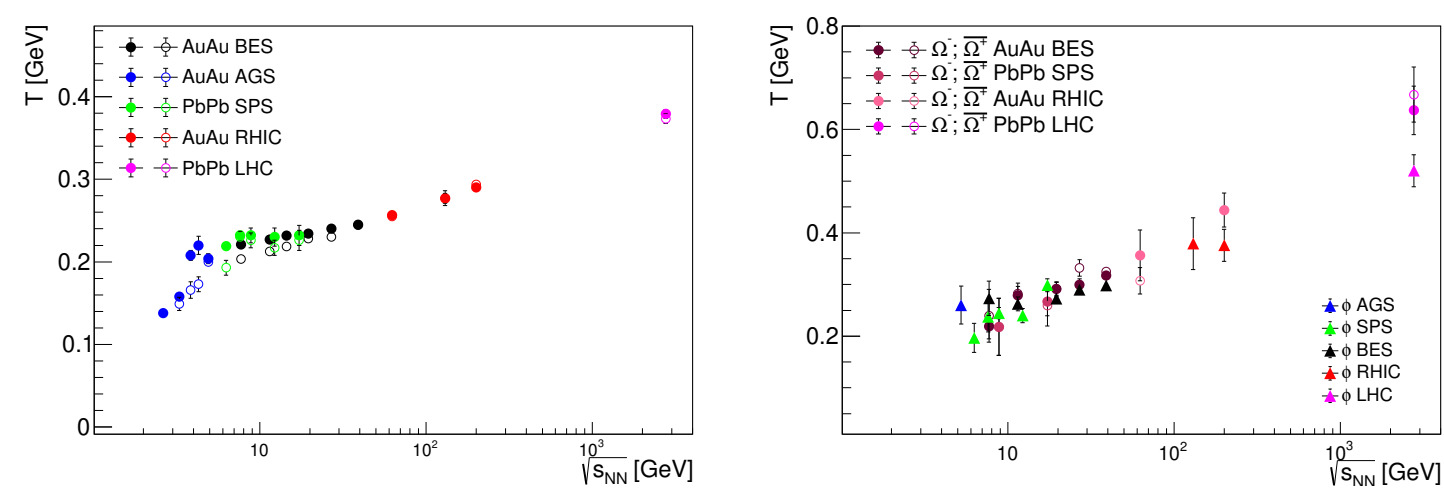

Figure 1: Energy dependence of the effective temperature of $K^{+}, K^{-}$(left) and $\Omega, \phi$ (right) in most central (0-5\% centrality) Au+Au collisions at RHIC-BES energies (black symbols). Previous results obtained in $\mathrm{Au}+\mathrm{Au}$ collisions at AGS energies [6,10], $\mathrm{Pb}+\mathrm{Pb}$ collisions at SPS energies [7, 11, 16], Au+Au collisions at RHIC energies $[8,12]$ and $\mathrm{Pb}+\mathrm{Pb}$ collisions at $\sqrt{s_{N N}}=2.76 \mathrm{TeV}[9,18]$ are also included.
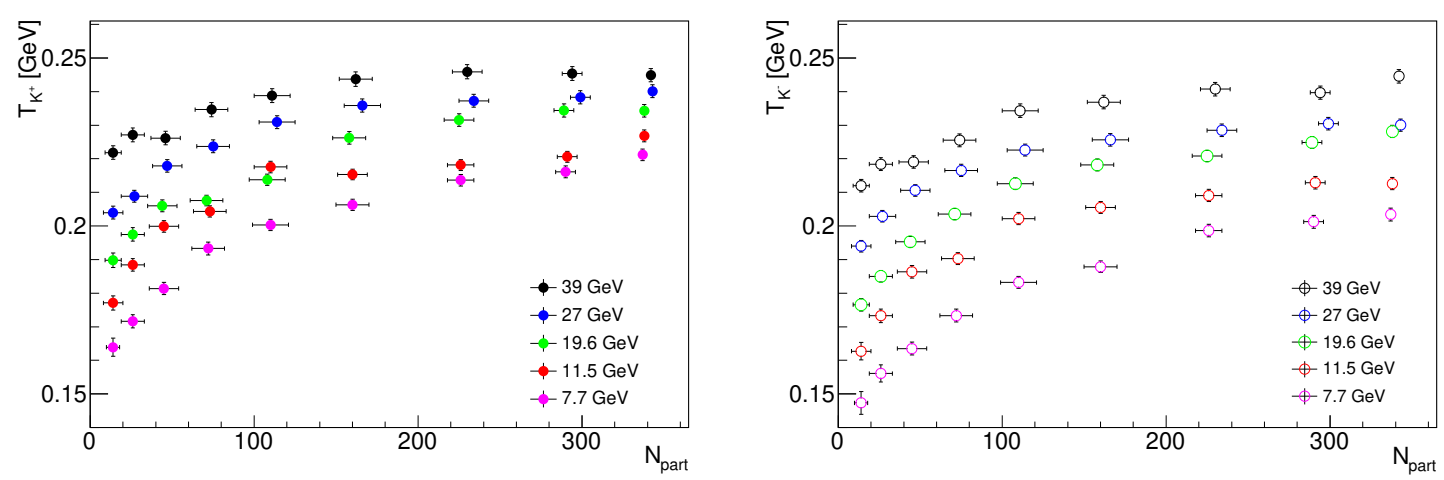

Figure 2: Effective temperature of $K^{+}$(left) and $K^{-}$(right) as a function of number of participants, $N_{\text {part }}$, in $\mathrm{Au}+\mathrm{Au}$ collisions at $\sqrt{s_{N N}}=7.7,11.5,19.6,27$ and $39 \mathrm{GeV}$.

For the same collision energy, the positive kaon $T$ is larger than the negative kaon $T$ for all centrality classes. However, the difference between the two temperatures for the same centrality decrease with the increasing energy. Therefore, the shape of $p_{T}$ spectra of $K^{+}$and $K^{-}$is different at lower RHIC-BES energies and becomes similar for higher energies. These results indicate the change of the kaon production mechanisms with energy. At lower energies the associated production dominates, while at higher energies there is an increased contribution to kaon production due to pair production, therefore the shape of $p_{T}$ spectra and corresponding inverse slopes become similar.

\section{Conclusions}

In this work, the effective temperature of $K^{ \pm}$produced in Au-Au collisions at RHIC-BES energies as a function of available energy and collision centrality was presented. In the lower RHIC-BES energy range, the $K^{+}$and $K^{-}$effective temperatures are almost constant from 7.7 to 19.6 GeV. This behavior, similar within error bars to what was observed at SPS energies, could 
indicate the onset of the deconfinement at these energies. The $T_{\phi}$ and $T_{\Omega}$ inverse slope parameters increases with the energy showing no plateau-like behaviour. The SPS and BES results are consistent within errors and no plateau-like behaviour is evidenced by the studied data.

\section{Acknowledgements}

This work was supported by IFA, FAIR-RO program, project number FAIR-08/2020.

\section{References}

[1] F. Karsch, Nucl. Phys. A 698 (2002) 199c

[2] J. Rafelski, B. Muller, Phys. Rev. Lett. 48 (1982) 1066.

[3] L. Adamczyk et al., STAR Collaboration, Phys. Rev. C 96 (2017) 44904; J. Adam et al., STAR Collaboration, Phys. Rev. C 101 (2020) 024905

[4] L. Adamczyk et al, STAR Collaboration, Phys. Rev. C 93 (2016) 021903

[5] M. Gazdzicki, M. Gorenstein, P. Seyboth, Acta Phys. Polon. B 42 (2011) 307

[6] L. Ahle et al., E866 and E917 Collaborations, Phys.Lett. B 490 (2000) 53; L. Ahle et al., E866 and E917 Collaborations, Phys.Lett. B 476 (2000) 1

[7] C. Alt et al., NA49 Collaboration, Phys. Rev. C 77 (2008) 024903; S. V. Afanasiev et al., NA49 Collaboration, Phys. Rev. C 66 (2002) 054902

[8] I. Arsene et al., BRAHMS Collaboration, Phys. Lett. B 687 (2010) 36; C. Adler et al., STAR Collaboration, Phys. Lett. B 595 (2004) 143; S. S. Adler et al., PHENIX Collaboration, Phys. Rev. C 69 (2004) 034909

[9] B. Abelev et al., ALICE Collaboration, Phys. Rev. C 88 (2013) 044910

[10] B. B. Back et al, E917 Collaboration, Phys. Rev. C 69 (2004) 054901

[11] C. Alt et al, NA49 Collaboration, Phys. Rev. C 78 (2008) 044907

[12] C. Adler et al., STAR Collaboration, Phys. Rev. C 65 (2002) 041901(R); S. S. Adler et al., (PHENIX Collaboration), Phys. Rev. C 72 (2005) 014903

[13] B. Abelev et al., (ALICE Collaboration), Phys. Rev. C 91 (2015) 024609

[14] J. Adams et al., (STAR Collaboration), Phys. Rev. Lett. 98 (2007) 062301

[15] M. M. Aggarwal et al., (STAR Collaboration), Phys. Rev. C 83 (2011) 024901

[16] C. Alt et al., (NA49 Collaboration), Phys. Rev. Lett 94 (2005) 192301

[17] B. Abelev et al., (ALICE Collaboration), Phys. Lett. B 728 (2014) 216

[18] O. Ristea, C. Ristea, A. Jipa, Int. J. Mod. Phys. E 29 (2020) 02, 2050009

[19] U. Heinz, R. Snellings, Ann. Rev. Nucl. Part. Sci. 63 (2013) 123 